\title{
Detection of Congestive Heart Failure using Renyi Entropy
}

\author{
David J Cornforth ${ }^{1}$, Herbert F Jelinek ${ }^{2}$ \\ ${ }^{1}$ University of Newcastle, Newcastle, Australia \\ ${ }^{2}$ Charles Sturt University, Albury, Australia
}

\begin{abstract}
Congestive Heart Failure (CHF) is a disease caused by the inability of the heart to supply the needs of the body in terms of oxygen and perfusion. Detection and diagnosis of $\mathrm{CHF}$ is difficult and requires a battery of tests, which include the electrocardiogram (ECG). Automated processing of the ECG signal and in particular heart rate variability (HRV) analysis holds great promise for diagnosis of CHF and more generally in assessing cardiac health, especially for personalized mobile health. However, recording the full 12-lead ECG is a relatively invasive procedure and for that reason it is of interest to determine what can be deduced from the much less intensive measurement of heart rate ( $R R$ interval) alone. In addition to calculating $S D N N$ and, RMSSD, which when combined gave an accuracy of $78.8 \%$ with the Nearest Neighbour classifier. The best Renyi entropy result was an accuracy of $66.7 \%$ using Nearest Neighbour. Combining the best Renyi entropy results with SDNN and RMSSD led to an overall accuracy of $87.9 \%$ with sensitivity of $80 \%$ and specificity of $94.4 \%$. In this work we have shown that applying Renyi entropy in addition to standard time domain measures identified CHF with higher accuracy than using time domain measures only. In addition, Renyi entropy exponents provide further information about the time signal characteristics that may be important in clinical decision making.
\end{abstract}

\section{Introduction}

Congestive Heart Failure (CHF) is a disease characterised by the inability of the heart to supply the needs of the body in terms of oxygen and perfusion leading to either compensated or uncompensated heart failure depending on the contribution of the sympathetic branches of the autonomic nervous system [1]. Identification of CHF is a complex process that includes medical imaging and 12-lead ECG, which are both relatively invasive. However a simple 3-lead ECG has also provided clinical relevant results combined with
HRV analysis [2]. Many different measures and guidelines have been developed for automated procedures that quantify the variability of the heart rate over time (HRV). These may be conveniently divided into time domain, frequency domain and nonlinear measures [3]. In the latter category, entropy measures quantify the degree of randomness in a system. Such measures include Shannon entropy, sample entropy, and Renyi entropy $[4,5,6,7]$. Each method has trade-offs relating to accuracy, linearity, and processing time. Several of the entropy measures may be extended to form multiscale measures, where analysis is performed over different scales or using different exponents to emphasize large or small values of the RR intervals. In previous work, we have shown the superior accuracy of the Renyi entropy to differentiate cardiac autonomic neuropathy from control, compared to other measures [8]. In addition, Renyi entropy is able to be applied to both different scales and different exponents. Renyi entropy $\mathrm{H}$, is a generalized measure, and includes the Shannon entropy as a special case [9]:

$$
H(\alpha)=\frac{1}{1-\alpha} \log _{2}\left(\sum_{i=1}^{n} p_{i}^{\alpha}\right)
$$

where $p_{i}$ is the probability that a random variable takes a given value out of $n$ values, and $\alpha$ is the order of the entropy measure. $H(0)$ provides the logarithm of $n$. As $\alpha$ increases the result becomes more sensitive to values occurring at higher probability. By using a selection of values for $\alpha$, a picture of the RR length distribution within a signal can be obtained, and this collection of measures can provide insight into the disease being studied.

In the implementation used here, the probability $p_{i}$ is estimated from the RR interval data by considering each sequence of $m$ RR intervals as a point in $m$-dimensional space, and adding the contribution of all other sequences using a Gaussian kernel method [8].

\section{Methods}

Data were obtained from the PhysioNet collection and comprised 18 patients with Normal Sinus Rhythm (NSR) and 15 patients with CHF. From each recording, 1000 RR 
(approx. 13 minutes) intervals were chosen from the middle of the recording to avoid any start-up and ending artefacts. The Renyi entropy was calculated using exponents $0<\alpha<5$, and with sequence length $\mathrm{I}=1$, 2, 4, 8 and 16. The Mann-Whitney test was used to determine the probability of the median of NSR and CHF being the same $(p<0.05)$.

We applied 5 machine learning algorithms from the Weka toolbox [10] including Naïve Bayes, Sequential minimal optimization (SMO), Nearest Neighbour, Decision Table and Decision Tree to this dataset, and used SDNN, RMSSD and Renyi entropy as input measures.

Naïve Bayes [11] estimates prior probabilities for each class by calculating simple frequencies of the occurrence of each value of each measure, given the class, then returning a probability of each class for unseen records.

Sequential Minimal Optimization (SMO) is based on the Support Vector Machine (SVM), which identifies records that define the boundaries of the different classes. SMO builds on this using polynomial kernels [12].

Nearest Neighbour [13] stores example records during training. An unlabeled record is compared to all records in the training set, using a distance measure, and the closest record is used to assign a class label.

Decision Table divides the dataset into cells, where each cell contains identical records. A record with unknown class is assigned the majority, or most frequent, class represented in the cell [14].

Decision Tree uses a version of the C4.5 decision tree algorithm [15], known as J48. Each measure is split using information gain, forming a branching structure. Subsequent splits are used until leaf nodes are formed, which contain only members of the same class. Unknown records are labeled by following the tree until a leaf node is reached.

Statistics included nonparametric group comparisons applying the Mann-Whitney test with p set as significant below 0.05 .

\section{Results}

A comparison of NSR with CHF using standard deviation (SDNN) for the two classes provided a significant p-value of 0.00039 from a Mann-Whitney test. The probability density functions for the two classes are shown graphically in Figure 1. This figure shows that the two classes are well separated by this measure, as the peak of the probability density curve for NSR occurs at the minimum for the curve representing CHF. This is as expected and supports previous results showing the efficacy of using SDNN to separate between these classes [16]. RMSSD was also examined to separate the classes, and a Mann-Whitney test provided a p-value of 0.00129 .

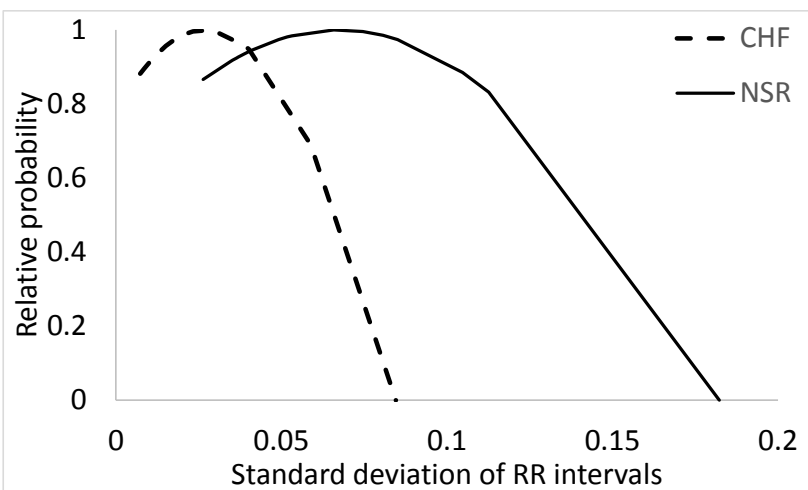

Figure 1. Graphical illustration of the class separation obtained for the standard deviation.

A comparison of NSR with CHF using Renyi coefficients is summarised in Table 1. Results are tabulated with values of $\alpha$ vertically increasing from 1 to 5 , and values of $m$ increasing from left to right. The pvalues for a sequence length of 8 are the lowest, suggesting a better separation of NSR from CHF using Renyi entropy calculated from sequences of 8 RR intervals. The lowest p-value of 0.0092 was obtained using $\alpha=3$. Several low p-values were also found using a sequence length of 16 , the lowest one being 0.03 , obtained with $\alpha=4$. These values suggest that Renyi entropy is able to distinguish between the classes of NSR and CHF, but the $\mathrm{p}$ values are higher than the value obtained using SDNN above.

Out of these Renyi entropy results, two with the lowest $\mathrm{p}$-value are selected for further illustration. These are for $m=8$ and for $m=16$. Figure 2 shows the probability density functions for NSR and CHF using Renyi entropy with $m=8$ and $\alpha=3$. Although the classes are clearly separated, as indicated by a p-value of 0.0092, the separation is not at the same level as shown in Figure 1. Figure 3 shows the probability density functions for NSR and CHF using Renyi entropy with $m=16$ and $\alpha=4$. Here the classes are also well separated, consistent with the p-value of 0.03, but not at the same level as shown in Figure 1 for SDNN. However both figures support the result from Table 1 that these measures are valuable for separating the classes.

TABLE 1. P-VALUES COMPARING NSR WITH CHF BASED ON RENYI ENTROPY. SIGNIFICANT RESULTS $(\mathrm{P}<0.05)$ ARE SHOWN IN BOLD.

\begin{tabular}{|c|c|c|c|c|c|}
\hline & \multicolumn{5}{|c|}{ Sequence Length } \\
\hline$a$ & 1 & 2 & 4 & 8 & 16 \\
\hline 1 & 0.800 & 0.914 & 0.233 & $\mathbf{0 . 0 1 9}$ & 0.159 \\
\hline 2 & 1.000 & 0.857 & 0.219 & $\mathbf{0 . 0 1 5}$ & 0.096 \\
\hline 3 & 0.857 & 0.857 & 0.294 & $\mathbf{0 . 0 0 9}$ & $\mathbf{0 . 0 4 7}$ \\
\hline 4 & 0.800 & 0.914 & 0.294 & $\mathbf{0 . 0 1 5}$ & $\mathbf{0 . 0 3 0}$ \\
\hline 5 & 0.772 & 0.885 & 0.347 & $\mathbf{0 . 0 2 1}$ & $\mathbf{0 . 0 3 3}$ \\
\hline
\end{tabular}


In previous work with cardiac autonomic neuropathy, we have shown the efficacy of Renyi entropy as a complimentary measure to SDNN, adding additional information [16]. In this work, we explored this for the CHF data set by first determining the correlation between SDNN and Renyi coefficient, and then using a machine learning study.

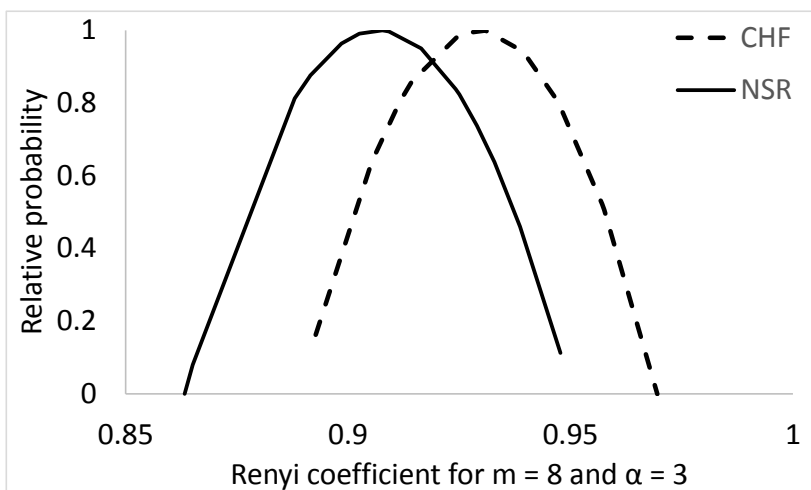

Figure 2. Graphical illustration of the class separation obtained for the Renyi entropy with $\mathrm{m}=8$ and $\alpha=3$.

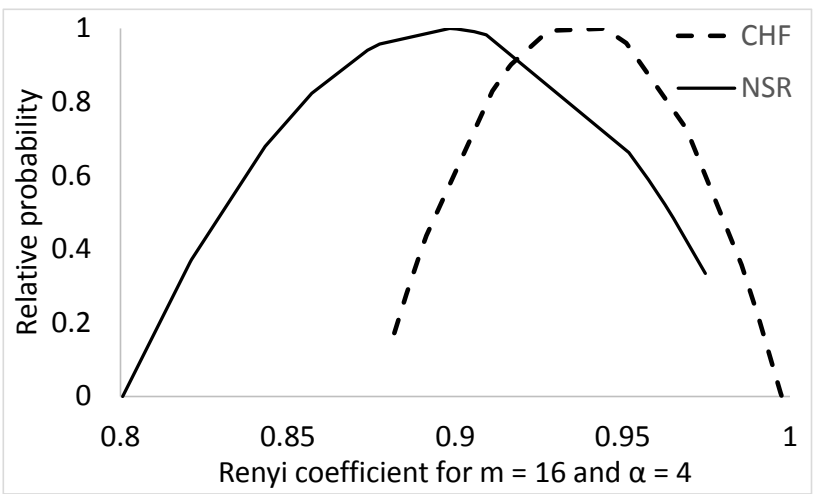

Figure 3. Graphical illustration of the class separation obtained for the Renyi entropy with $\mathrm{m}=16$ and $\alpha=4$.

In order to illustrate the effects of combining more than one measure, we now show results from a correlation study. Figure 4 shows the scatter plot of Renyi entropy with $m=8$ and $\alpha=3$ versus SDNN. The plot indicates that the two classes are interleaved. This means that either measure, by itself, cannot perfectly separate the classes, because one measure can only separate using a line drawn perpendicular to either the $\mathrm{x}$-axis or $\mathrm{y}$-axis. However, by combining two measures in this case, both measures contribute towards separation of the classes. For example, a simple classification scheme could now, in effect, work by drawing a diagonal line to separate classes, and so would achieve a better separation. Drawing such a line can be optimally performed using an automated classification algorithm.

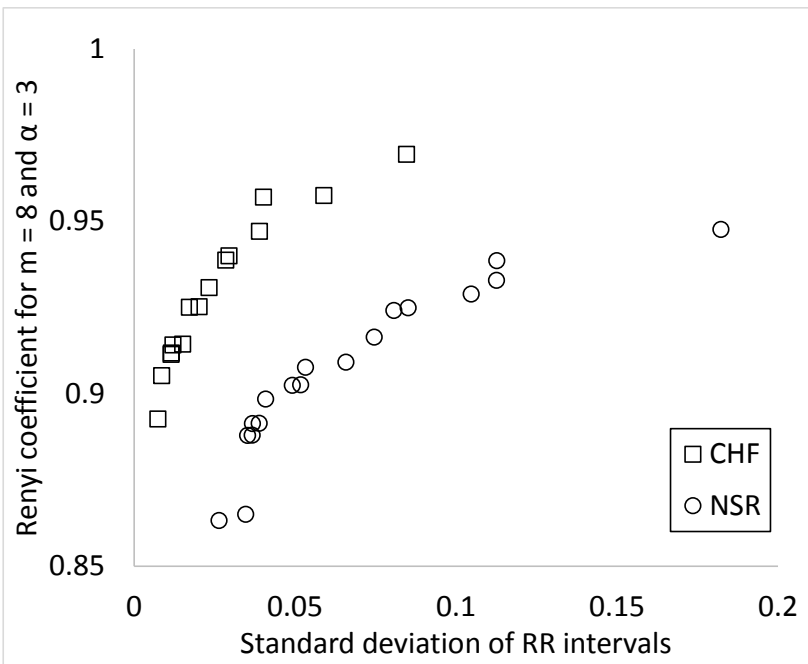

Figure 4. Scatter plot showing relationship between the Renyi entropy with $m=8$ and $\alpha=3$ and the standard deviation of RR intervals.

As the classes are obviously interleaved, as illustrated in Figure 4, this application would be better served by utilizing a machine learning algorithm to provide an improved separation. A further advantage of this is that such an algorithm is capable of using not only two measures as in Figure 4, but any number. Results from the classifier algorithms applied and their accuracy are now described.

Table 2 shows results from classification algorithms using only two variables: SDNN and RMSSD. The best result was $78.8 \%$ accuracy using Nearest Neighbour with sensitivity of $73.3 \%$. This needs improvement before considering time domain measures alone as suitable for accurate identification of CHF.

TABLE 2. RESULTS FROM AUTOMATED CLASSIFICATION USING ONLY SDNN AND RMSSD.

\begin{tabular}{|l|l|l|l|}
\hline Classifier & Sens & Spec & Acc \\
\hline Naïve Bayes & $86.7 \%$ & $61.1 \%$ & $72.7 \%$ \\
\hline SMO & $46.7 \%$ & $77.8 \%$ & $63.6 \%$ \\
\hline Nearest Neighbour & $73.3 \%$ & $83.3 \%$ & $78.8 \%$ \\
\hline Decision Table & $53.3 \%$ & $88.9 \%$ & $72.7 \%$ \\
\hline Decision Tree & $53.3 \%$ & $83.3 \%$ & $69.7 \%$ \\
\hline
\end{tabular}

Table 3 shows results using only the two best Renyi coefficients identified from Table 1 , namely $\mathrm{H}(8,3)$ and $\mathrm{H}(16,4)$. Here the best accuracy obtained was $66.7 \%$. Although this is consistent with the above analysis, where the Renyi coefficients provide a good separation between classes, it does not match that provided by SDNN and again used as is cannot be used for diagnostic $\mathrm{CHF}$ identification. 
TABLE 3. RESULTS FROM AUTOMATED CLASSIFICATION USING ONLY $\mathrm{H}(8,3)$ AND $\mathrm{H}(16,4)$.

\begin{tabular}{|l|l|l|l|}
\hline Classifier & Sens & Spec & Acc \\
\hline Naïve Bayes & $60.0 \%$ & $66.7 \%$ & $63.6 \%$ \\
\hline SMO & $53.3 \%$ & $77.8 \%$ & $66.7 \%$ \\
\hline Nearest Neighbour & $46.7 \%$ & $66.7 \%$ & $57.6 \%$ \\
\hline Decision Table & $20.0 \%$ & $55.6 \%$ & $39.4 \%$ \\
\hline Decision Tree & $66.7 \%$ & $27.8 \%$ & $45.5 \%$ \\
\hline
\end{tabular}

Table 4 shows results from classification algorithms when the two types of measurements are combined. The time domain measures SDNN and RMSSD are combined with the Renyi coefficients $H(8,3)$ and $H(16,4)$. The result is that classification is much improved, with a maximum accuracy of $87.9 \%$ using the Nearest Neighbour classifier, and good sensitivity of $80 \%$ and specificity of $94.4 \%$.

TABLE 4. RESULTS FROM AUTOMATED CLASSIFICATION USING SDNN RMSSD, $\mathrm{H}(8,3)$ AND $\mathrm{H}(16,4)$.

\begin{tabular}{|l|l|l|l|}
\hline Classifier & Sens & Spec & Acc \\
\hline Naïve Bayes & $80.0 \%$ & $66.7 \%$ & $72.7 \%$ \\
\hline SMO & $66.7 \%$ & $77.8 \%$ & $72.7 \%$ \\
\hline $\begin{array}{l}\text { Nearest } \\
\text { Neighbour }\end{array}$ & $80.0 \%$ & $94.4 \%$ & $87.9 \%$ \\
\hline Decision Table & $53.3 \%$ & $88.9 \%$ & $72.7 \%$ \\
\hline Decision Tree & $53.3 \%$ & $83.3 \%$ & $69.7 \%$ \\
\hline
\end{tabular}

\section{Conclusion}

Identification of cardiac pathology including CHF is becoming more of an interest to lay people requiring simple, accurate and suitable ways of providing information. HRV is a simple way of characterising structural attributes of a time series. The information from a 3-lead ECG is much easier to obtain that a full ECG and can be provided by many commercial monitors. Heart rate alone can be provided by some smart watches, so is accessible to the layperson. The current work shows a high degree of accuracy in identifying CHF from short 3lead ECG recordings and suggests that Renyi entropy can enhance the performance of automated diagnostic tools above the performance when using only time domain measures. Renyi entropy provides extra accuracy and the method of using both time domain and nonlinear measures makes it is feasible to add to personalized home analysis of short ECG recordings.

\section{Acknowledgements}

The authors thanks Bev de Jong for technical assistance in recording of ECGs, and Roche Australia for providing Glucose measuring kits.

\section{References}

[1] C. Tissot, E. M. da Cruz, S. D. Miyamoto. Congestive Heart Failure. In Da Cruz, M. Eduardo (eds) Pediatric and Congenital Cardiology, Cardiac Surgery and Intensive Care, 2013 pp 20452062, isbn="978-1-4471-4619-3", doi="10.1007/978-1-44714619-3_229", $\quad$ url=http://dx.doi.org/10.1007/978-1-4471-4619$\underline{3229}$

[2] Tsuji, H., M. G. Larson, F. J. Venditti, E. S. Manders, J. C. Evans, C. L. Feldman and D. Levy (1996). "Impact of reduced heart rate variability on risk for cardiac Events: The Framingham Heart Study." Circulation 94(11): 2850-2855.

[3] TFESC/NASPE, "Heart rate variability. Standards of measurement, physiological interpretation, and clinical use. Task Force of the European Society of Cardiology and the North American Society of Pacing and Electrophysiology," European Heart Journal, vol. 17, pp. 354-381, 1996.

[4] J. R. Pacheco, D. T. Román, and H. T. Cruz, "Distinguishing stationary/nonstationary scaling processes using wavelet Tsallis qentropy," Mathematical Problems in Engineering, p. 867042, 2012.

[5] D. Cysarz, H. Bettermann, and P. van Leeuwen, "Entropies of short binary sequences in heart period dynamics," Am J Physiol Heart Circ Physiol, vol. 278, pp. H2163-2172, June 1, 20002000.

[6] M. Costa, A. L. Goldberger, and C.-K. Peng, "Multiscale entropy analysis of biological signals," Physical Review E, vol. 71, 2005.

[7] M. Baumert, V. Baier, J. Haueisen, N. Wessel, U. Meyerfeldt, A. Schirdewan, and A. Voss, "Forecasting of life threatening arrhythmias using the compression entropy of heart rate," Methods of Information in Medicine, vol. 43, pp. 202-6, 2004.

[8] Cornforth D, Jelinek HF, Tarvainen M, 'A Comparison of Nonlinear Measures for the Detection of Cardiac Autonomic Neuropathy from Heart Rate Variability', ENTROPY, 17 14251440 (2015)

[9] A. Rényi, "On measures of information and entropy," Proceedings of the fourth Berkeley Symposium on Mathematics, Statistics and Probability, pp. 547-561, 1960.

[10] Witten, I.H., and Frank, E. (2005): Data Mining: Practical Machine Learning Tools and Techniques with Java Implementations, Morgan Kaufmann, San Francisco, 2005.

[11] Bayes, T. An essay towards solving a problem in the doctrine of chances. Philos Trans R Soc Lond 1763;53:370-418.

[12] Platt J. Fast Training of Support Vector Machines using Sequential Minimal Optimization. In Schoelkopf B, Burges C, Smola A. editors. Advances in Kernel Methods - Support Vector Learning, MIT Press 1998.

[13] Fisher RA. The use of multiple measurements in taxonomic problems, Annu Eugen 7(II) 1936:179-188 (Reprinted in Contributions to Mathematical Statistics, Wiley, 1950).

[14] Kohavi R. The power of decision tables. In Proc Eur Conf Machine Learn, Lect Notes Art Intelligence 914, Springer Verlag, 1995: 174-189.

[15] Quinlan JR. Induction of decision trees. Mach Learn 1986;1(1):81-106.

[16] Cornforth DJ, Tarvainen MP, Jelinek HF, 'Evaluation of normalised Renyi entropy for classification of cardiac autonomic neuropathy', 2014 8th Conference of the European Study Group on Cardiovascular Oscillations, ESGCO 2014 (2014).

Address for correspondence.

David Cornforth.

Applied Informatics Research group,

University of Newcastle, Callaghan 2308 NSW Australia David.Cornforth@newcastle.edu.au 